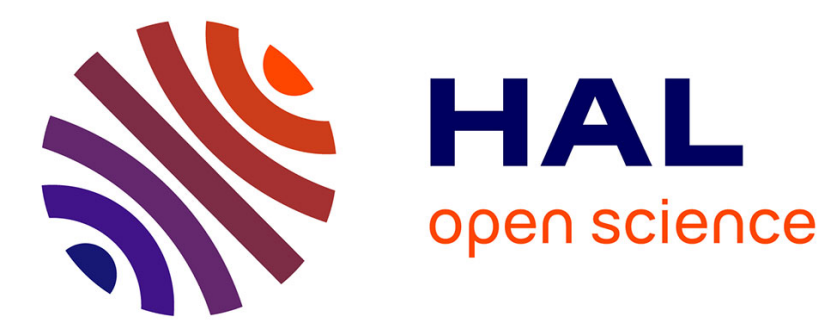

\title{
Logarithmic velocity variations for longitudinal ultrasonic waves in cubic BaTio3 near its ferroelectric transition
}

\author{
J.M. Courdille, J. Dumas, J. Servais
}

\section{To cite this version:}

J.M. Courdille, J. Dumas, J. Servais. Logarithmic velocity variations for longitudinal ultrasonic waves in cubic BaTio3 near its ferroelectric transition. Journal de Physique Lettres, 1978, 39 (13), pp.227230. 10.1051/jphyslet:019780039013022700 . jpa-00231485

\section{HAL Id: jpa-00231485 https://hal.science/jpa-00231485}

Submitted on 1 Jan 1978

HAL is a multi-disciplinary open access archive for the deposit and dissemination of scientific research documents, whether they are published or not. The documents may come from teaching and research institutions in France or abroad, or from public or private research centers.
L'archive ouverte pluridisciplinaire HAL, est destinée au dépôt et à la diffusion de documents scientifiques de niveau recherche, publiés ou non, émanant des établissements d'enseignement et de recherche français ou étrangers, des laboratoires publics ou privés. 


\title{
Classification \\ Physics Abstracts \\ $77.80-62.65-64.70 \mathrm{~K}-43.35$ \\ LOGARITHMIC VELOCITY VARIATIONS \\ FOR LONGITUDINAL ULTRASONIC WAVES IN CUBIC BaTiO ${ }_{3}$ NEAR $^{2}$ ITS FERROELECTRIC TRANSITION $+$
}

\author{
J. M. COURDILLE, J. DUMAS and J. SERVAIS
}

Laboratoire d'Ultrasons $\left({ }^{*}\right)$, Université Pierre-et-Marie-Curie, Tour 13, 4, place Jussieu, 75230 Paris Cedex 05, France

(Reçu le 29 mars 1978, révisé le 19 mai 1978, accepté le 22 mai 1978)

\begin{abstract}
Résumé. - Les variations de vitesse et d'atténuation ultrasonores ont été mesurées au voisinage de la transition ferroélectrique dans $\mathrm{BaTiO}_{3}$. Aucun comportement critique n'est observé sur l'atténuation tandis que les constantes élastiques varient selon $\log \left(T-T_{0}\right)$. Ces résultats ne semblent pas pouvoir être expliqués d'une manière satisfaisante à l'aide des hypothèses utilisées précédemment à propos de K.T.N.
\end{abstract}

\begin{abstract}
Ultrasonic velocity and attenuation measurements have been made in the vicinity of the ferroelectric transition in $\mathrm{BaTiO}_{3}$. The attenuation does not show any critical behaviour, and the velocity variation has a $\log \left(T-T_{0}\right)$ dependence. No satisfactory hypothesis can be put forward to explain both these $\mathrm{BaTiO}_{3}$ measurements and the previous results on K.T.N.
\end{abstract}

1. Introduction. - The study of phase transitions by means of high-frequency ultrasonic waves gives information about the dynamical properties of the order parameter and about the coupling between the strain and the order parameter fluctuations [1]. Using the linear response theory [2], one can predict very well defined values for the critical exponents of ultrasonic velocity and attenuation; these last two quantities are very sensitive functions with regard to the anisotropy of the soft-mode dispersion curves, inside the Brillouin zone : this is well illustrated by the experimental and theoretical results obtained in studies of A.D.P. [3], $\mathrm{KTa}_{x} \mathrm{Nb}_{1-x} \mathrm{O}_{3}$ [2], and $\mathrm{VO}_{2}$ [4], for examples.

$\mathrm{BaTiO}_{3}$, one of the most typical ferroelectric crystals, has many properties which are not quite elucidated at this time. As it is possible at present to obtain large $\left(\simeq 1 \mathrm{~cm}^{3}\right)$ single crystals of good crystallographic quality, we have studied the high temperature phase transition of $\mathrm{BaTiO}_{3}$ by the ultrasonic method. As a matter of fact the available results are incomplete $[5,6]$ and often restricted to a small range of temperature. Our measurements show that the behaviour of $\mathrm{BaTiO}_{3}$ in its cubic phase $(\mathrm{Pm} 3 \mathrm{~m})$ cannot

$\left({ }^{*}\right)$ Associated with the Centre National de la Recherche Scientifique. be explained directly with the dispersion laws which are customary ușed in the case of the perovskites.

We present successively :

- the experimental results and their analysis ;

- a recall of the theoretical results achieved in the frame of the mean field approximation, for the different dispersion curves ;

- a discussion of different hypothesis which could be put forward to explain our puzzling result : none of them is able to give a complete explanation of the experimental results and also to be in agreement with the K.T.N. ultrasonic results [2].

2. Experimental results. $-\mathrm{BaTiO}_{3}$ is a cubic perovskite $(\mathrm{Pm} 3 \mathrm{~m})$ at high temperatures and shows in the vicinity of $130^{\circ} \mathrm{C}$ a ferroelectric phase transition to a tetragonal structure [7]. We used single domain samples extracted from a $\mathrm{BaTiO}_{3} \operatorname{crystal}\left(T_{\mathrm{c}} \simeq 130^{\circ} \mathrm{C}\right)$ provided by Sanders Associates. We measured in the non-polar phase of $\mathrm{BaTiO}_{3}$ the temperature variations of velocity and attenuation of an ultrasonic longitudinal wave propagating along [1.0.0] axis at the frequency of $540 \mathrm{MHz}$. The method of measurements and the experimental set up are quite classical and have been described elsewhere [8].

Results are given in figures 1 and 2 . The velocity, corresponding to the elastic constant $C_{11}$ - 


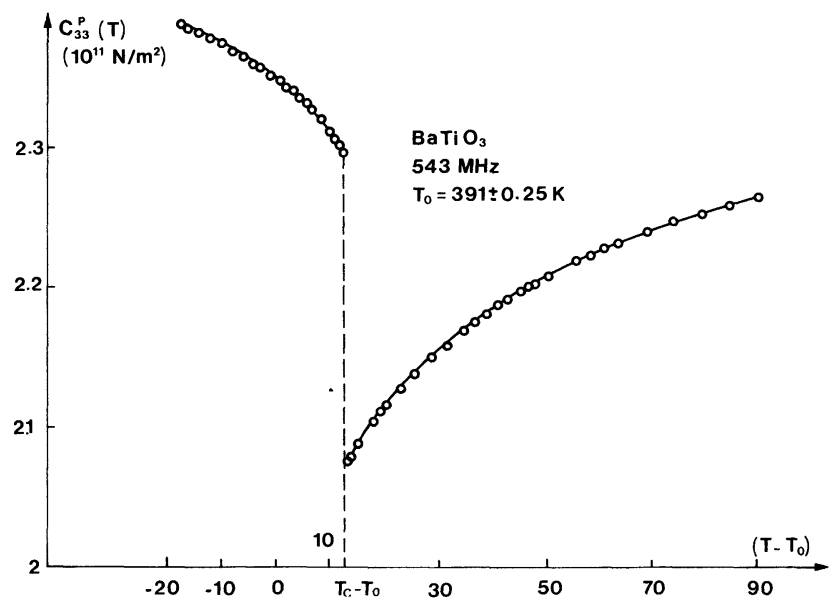

FIG. 1. - Velocity variations of an ultrasonic longitudinal wave (540 $\mathrm{MHz}$ ) propagating along [1.0.0] axis in the vicinity of the ferroelectric transition in $\mathrm{BaTiO}_{3}$.

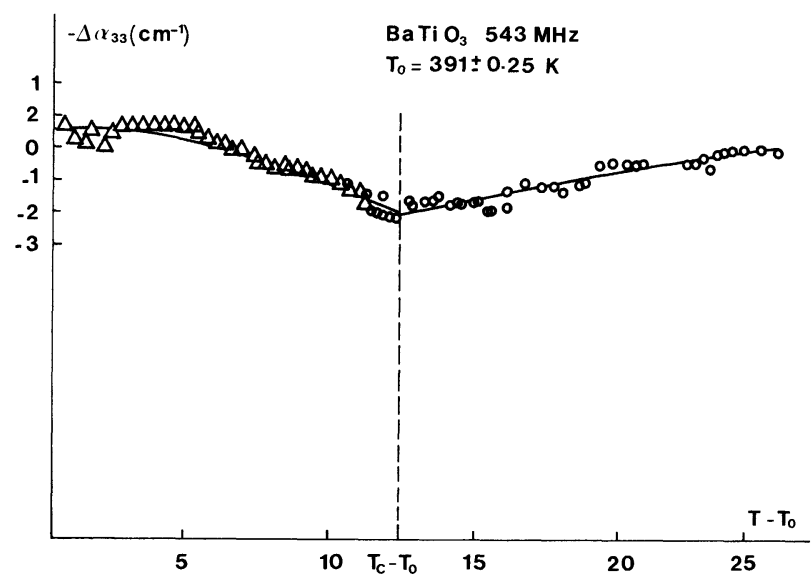

Fig. 2. - Ultrasonic attenuation of a longitudinal wave propagating along [1.0.0] axis in the vicinity of the ferroelectric transition in $\mathrm{BaTiO}_{3}$.

$\left(C_{11}=C_{22}=C_{33}\right)$, decreases continuously from the highest temperature $\left(\simeq 220^{\circ} \mathrm{C}\right)$ down to the transition $\left(\simeq 130^{\circ} \mathrm{C}\right)$. On the contrary the attenuation does not show any critical variations. Let us observe that the ultrasonic path in the crystal is about $3 \mathrm{~mm}$ long. In these conditions we can rule out the influence of surface defects or strains on velocity variations : the perturbed zone does not affect more than some microns as we used a delicate method of polishing by hand, and could not have a significant influence. We checked the crystal for bulk strains with a polarizing microscope and we did not find any preferential direction in the cubic phase of our samples. To analyse the results we need to know the temperature $T_{0}$ with accuracy : the best way to obtain it is to plot the reciprocal dielectric constant $\varepsilon^{-1}$ as a function of temperature in the cubic phase. If we fit $\varepsilon^{-1}(T)$ with a straight-line we get the temperature $T_{0}$ equal to
$391 \pm 0.25 \mathrm{~K}$. Experimentally, this $T_{0}$ determination is achieved during ultrasonic measurements, in order to have reliable results. The reciprocal dielectric constant varies linearly in the cubic phase and we do not find any other behaviour approaching the transition. In particular, we do not observe any kind of dipolar coupling which would be detected by a different slope [9]. This result indicates that the measurements we present concern only the Landau or mean-field region of the transition; it is also a supplementary proof of the homogeneity of the samples we used.

We tried to fit the velocity variations with a function $A\left(T-T_{0}\right)^{-n}+B$ : such a law appears in the theoretical results, where the $n$ critical exponent depends on the soft mode dispersion surface dimensionality. The immediate and obvious result is that the fit is not possible for a large temperature range and gives near the transition a value far below 0.5 . So we tried a fit with a function $A \log \left(T-T_{0}\right)+B$ and we obtain a very good result over the whole temperature range (Fig. 3).

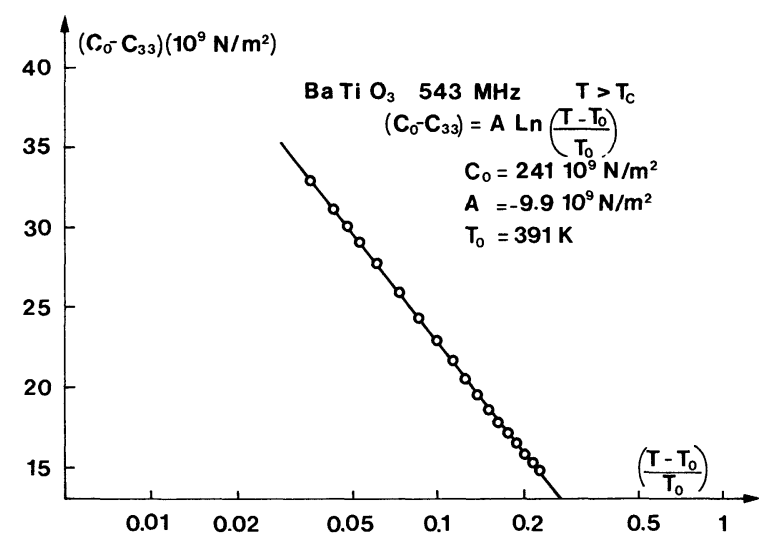

FIG. 3. - Fit of $\Delta C_{11}(T)=B-C_{11}(T)$ as a function of $\left(T-T_{0}\right) / T_{0}$ in a semi-Log diagram.

Unexpectedly, experimental results in $\mathrm{BaTiO}_{3}$ are very different from other ferroelectric perovskites like K.T.N. for example [2] : in this compound, both ultrasonic attenuation and velocity vary near the transition with critical exponents equal to 1.5 and 0.5 respectively, values which are in very good agreement with possible couples of exponents expected from the theory we mention eventually.

We are going to discuss in the next chapter this discrepancy between crystals the behaviour of which is expected to be nearly the same.

3. Theory. - 3.1. - The main features of the theory [10] used to explain ultrasonic experiments near phase transitions are :

- a mean field approximation;

- an electrostrictive coupling between strain and electrical polarization fluctuation ; 
- different models [2] of the soft mode dispersion curve depending on the dimensionality.

The frequency $\omega(q)$ of the soft mode dispersion curve is written for a phonon mode wave vector $\mathbf{q}$ $\left(q_{x}, q_{y}, q_{z}\right)$ :

$$
\begin{aligned}
\omega^{2}(q, T) & =\omega_{0}^{2}(T)+\Lambda_{1} q_{x}^{2}+\Lambda_{2} q_{y}^{2}+\Lambda_{3} q_{z}^{2} \\
\omega_{0}^{2}(T) & =A\left(T-T_{0}\right)
\end{aligned}
$$

$A, \Lambda_{1}, \Lambda_{2}, \Lambda_{3}$, are constants independent of $q$ and $T$.

$$
\text { If } \begin{aligned}
& \Lambda_{1} \simeq \Lambda_{2} \simeq \Lambda_{3}, \text { the dimensionality is } 3 ; \\
& \Lambda_{1} \simeq \Lambda_{2} \gg \Lambda_{3}, \text { the dimensionality is } 2 ; \\
& \Lambda_{1} \gg \Lambda_{2} \simeq \Lambda_{3}, \text { the dimensionality is } 1 .
\end{aligned}
$$

These three cases are respectively written

$$
\begin{aligned}
& \omega^{2}=\omega_{0}^{2}+\Lambda q^{2} \\
& \omega^{2}=\omega_{0}^{2}+\Lambda\left(q_{x}^{2}+q_{y}^{2}\right) \\
& \omega^{2}=\omega_{0}^{2}+\Lambda q_{x}^{2} .
\end{aligned}
$$

3.2 In the frame of this theory and with these dispersion curve models, the velocity and attenuation critical exponents are found to be very much dependent on the anisotropy but these values are never smaller than 0.5 and 1.5 respectively : a slight anisotropy in the dispersion curve can only lower the dimensionality and both values of the critical exponents increase accordingly. Some variations of these models can be proposed to obtain a lower temperature dependence than $\left(T-T_{0}\right)^{-1 / 2}$.

- A dispersion law $\omega_{0}^{2}+\Lambda q^{4}$ takes into account higher order terms in wave vector and increases less rapidly than a quadratic function for small $q$ : the calculated critical exponents are always greater than $\frac{5}{4}$. The introduction of higher order terms in the dispersion law does not lower the $n$-valueat all.

- The $\omega_{0}^{2}+\Lambda q^{2}$ model is, strictly speaking, valid only for an isotropic medium : the cubic symmetry can be taken into account by $\Lambda=\Lambda(\theta, \varphi)$ where $\theta$ and $\varphi$ are spherical angular coordinates [11]. The velocity and attenuation have the same temperature variations with this correction than in the isotropic case : the theoretical values differ only by a factor of the order of 1 [12].

- Anomalous diffusion in X-ray experiments [13] has been observed in $\mathrm{BaTiO}_{3}$ cubic phase : the characteristics vary very slowly with the temperature. The proposed explanation is based on displacement correlations along the [1.0.0], [0.1.0], [0.0.1] axes of the cubic structure. This chain model can explain, in particular, the Raman spectrum measured in the
$\mathrm{BaTiO}_{3}$ cubic phase [14]. For us, the main feature we select is the existence of an uniaxial order in the cubic phase.

Levanyuk [15] computed the ultrasonic velocity behaviour in an uniaxial system, with a dispersion law

$$
\omega^{2}(q)=\omega_{0}^{2}+\Lambda q^{2}+\alpha \cos ^{2} \theta
$$

( $\theta$ is the angle between the wave vector $\mathbf{q}$ and the uniaxial axis). The result is a $\log \left(T-T_{0}\right)$ variation. Such a behaviour has been experimentally found in T.G.S., for example [16].

It is tempting in these conditions to consider $\mathrm{BaTiO}_{3}$ in its cubic phase as a superposition of independent uniaxial objects - the correlation chains - and to explain our results by considering this microscopic feature : unfortunately an attenuation effect is also expected with this model and is not experimentally observed.

- An indirect mechanism of coupling can be put forward. An ultrasonic experiment gives a measurement of the adiabatic elastic constants $C_{11}^{S}$. On the contrary, the theory we mentionned earlier, gives an isothermal elastic constant $C_{11}^{T}$. A thermodynamic calculation shows $[17,12]$ that these two quantities are related by the specific heat $\mathrm{C}_{v}$.

In a mean field theory, it is possible to show in a similar way as for ultrasonic quantities that the part of the specific heat $\mathcal{C}_{v}$ which is related with soft-mode variation, has a behaviour characterized by critical indices $[18,19]$ depending on the dimensionality : but these indices are the same as for ultrasonic velocity.

This explanation by specific heat variations could only explain the lack of ultrasonic attenuation near the transition.

- The difference observed in the behaviour of the ultrasonic attenuation in $\mathrm{BaTiO}_{3}$ and K.T.N. [2], taken alone, could also be explained by numerical values of the electrostrictive coupling constants $g$. In $\mathrm{BaTiO}_{3}$ its value from [20] is near 3 c.g.s. In K.T.N., the value deduced from our ultrasonic experiments [2] is around 12 c.g.s. The $g$ values appear as the square in the calculation of the velocity and attenuation and this fact is sufficient to explain the difference in the attenuation pattern, but not to explain the logarithmic variations of the elastic constant.

4. Conclusion. - After having examined different possibilities to explain experimental results in both $\mathrm{BaTiO}_{3}$ and in K.T.N., we did not find any simple and reliable theoretical explanation for the logarithmic variation of velocity observed.

\section{References}

[1] Pytte, E., Phys. Rev. B 1 (1970) 924.

[2] Courdille, J. M., Dumas, J., Ziolkiewicz, S., Joffrin, J., J. Physique 38 (1977) 1519.
[3] Billmann, A., Levelut, A., J. Physique 38 (1977) 1307.

[4] Prieur, J.-Y., Seznec, P., Ziolkiewicz, S., J. Physique Lett. 38 (1977) L-25. 
[5] Kashida, S., Matta, I., Ikushima, A., J. Phys. Soc. Jpn 34 (1973) 997.

[6] Strukov, B. A., Garland, K. V., Sov. Phys. Crystallogr. 19 (1974) 175

[7] Megaw, H. D., Proc. Phys. Soc. Am. 58 (1946) 133.

[8] Bömmel, H. E., Dransfeld, D. K., Phys. Rev. Lett. 1 (1958) 234.

[9] Kind, R. and Müller, K. A., Commun. Phys. 1 (1976) 223.

[10] Dvorak, V., Czech. J. Phys. B 21 (1971) 836.

[11] Harada, J., Axe, J. D., Shirane, G., Phys. Rev. B 4 (1971) 155.

[12] Courdille, J. M.. Thèse, Université Paris VI, février 1978.

[13] Comes, R., Lambert, M., Guinier, A., C. R. Acad. Sci. Paris 266 (1968) 959.
[14] Quittet, A. M., Lambert, M., Solid State Commun. 12 (1973) 1053

[15] Levanyuk, A. P., Minaeva, K. A., Strukov, B. A., Sov. Phys. Solid State 10 (1969) 1919.

[16] Strukov, B. A., Kkhana, S. K., Minaeva, K. A., Sov. Phys. Solid State 18 (1976) 1934.

[17] Landau, L., Lifchitz, E. K., Théorie de l'Elasticité (Edition MIR), page 27.

[18] Levanyuk, A. P., Sov. Phys. Solid State 5 (1964) 1294.

[19] Levanyuk, A. P., Izv. Akad. Nauk S.S.S.R. Sci. Fiz. 29 (1965) 878 [Bull. Acad. Sc. Phys. Ser., page 885].

[20] Jona, F. and ShIRANE, in Ferroelectric Crystals (Pergamon Press) 1962, p. 108. 